\title{
PROBIOTIC COMPOSITION OF FERMENTED COW MILK AND SOY MILK EFFECT ON EPIDERMAL GROWTH FACTOR AND EPIDERMAL THICKNESS IN FEMALE WISTAR RATS
}

\author{
R. Lesmana ${ }^{1,2}$, L. Adriani ${ }^{3}$, Z. Haryawan ${ }^{4}$, H. Goenawan ${ }^{1,2}$, Y. S. Pratiwi ${ }^{1,2}$, N. Sylviana ${ }^{1,2}$ and U. Supratman ${ }^{5}$ \\ ${ }^{1}$ Physiology Division, Department of Biomedical Sciences, Faculty of Medicine, Universitas Padjadjaran, Jatinangor \\ 45363, Indonesia. \\ ${ }^{2}$ Molecular Laboratory, Biological Activity Division, Central Laboratory, Universitas Padjadjaran, Jatinangor 45363, \\ Indonesia. \\ ${ }^{3}$ Faculty of Animal Husbandry, Universitas Padjadjaran, Jatinangor 45363, Indonesia. \\ ${ }^{4}$ Undergraduate Program Medical Doctor, Faculty of Medicine, Universitas Padjadjaran, Jatinangor 45363, Indonesia. \\ ${ }^{5}$ Department of Chemistry, Faculty of Mathematics and Natural Sciences, Universitas Padjadjaran, Jatinangor 45363, \\ Indonesia. \\ *Correspondence author’s email: ronny@unpad.ac.id
}

\begin{abstract}
Isoflavone has an effect on countering the progression of skin aging, while probiotics boost the absorption of nutrients and maintain the skin tissue. Fermentation of isoflavone might be more beneficial for inducing cytokine system and boosting the skin regeneration. There is limited information about the difference of effect between the various formulation of fermented cow milk and soy milk towards the skin regeneration via epidermal growth factor (EGF) signaling. Twenty-five female rats were divided into 5 group for 12 weeks supplementation of fermented soy and cow milk by oral gavaging. Specific probiotics composition was utilized for fermenting formulation cow and soymilk consist (Lactobacillus bulgaricus, Streptococcus thermophiles, Lactobacillus acidophilus, and Bifidobacterium bifidum). Our data showed that there are different characteristics of stool and its macroscopic appearance after different probiotics composition of fermented milk treatment. Histology results showed that the epidermal skin thickness was found higher in every treatment group. Similar results are found from EGF protein levels. Fermented cow milk with Bifidobacterium bifidum and Lactobacillus acidophilus were found to have a thicker epidermal and higher level of EGF protein level. Milk fermented by Bifidobacterium bifidum showed the most significant effect in regenerating skin tissue. Therefore, it might prevent the skin aging process compared to the other formulations. Compared to conventional fermented milk, the composition of soy milk might enhance this positive effect of fermented milk on the skin.
\end{abstract}

Key words: Fermented milk, probiotics, isoflavone, regeneration, epidermal thickness

https://doi.org/10.36899/JAPS.2021.4.0316

Published online December 18, 2020

\section{INTRODUCTION}

Skin is the barrier that protects the body against water loss and microorganism infection. Like other tissues, skin also is affected by the aging process. Wrinkles and unwanted spots usually characterize skin aging. They are caused by decreasing skin elasticity due to the degradation of collagen, which reduces the mechanical tension of the tissue. The increased rate of collagen degradation creates a fragmented collagen structure (Fisher et al., 2014). Several growth factors are involved in the regulation of skin homeostasis. Among these, EGF and its receptor (EGFR) have a broad spectrum of effects including cell proliferation and differentiation. EGF facilitates the construction of skin tissue through dermal regeneration, proliferation, differentiation, and migration of keratinocyte, endothelial cells, and fibroblast through interaction with EGFR (Bodnar, 2013; Zeng and Harris 2014). Attachment and binding of EGF ligand to EGFR induces the dimerization of the receptors. Dimerization then phosphorylates the intracellular domain of tyrosine kinase that leads to activation of various downstream signaling pathways, including Ras/MAPK, PLC $\gamma 1 / \mathrm{PKC}$, PI3K/Akt, and STAT that lead to skin homeostasis (Wang, 2017; Rajaram et al., 2017).

These growth factors are known to have been found from food sources such as milk. Insulin-like growth factor (IGF-1), one of the most significant growth factors, have already been identified from various dairy products. Milk content was associated with higher levels of IGF-I and IGFBP3 levels (Srinivasan et al., 2019). Also, dietary modification of intestinal microflora by yogurt or Lactobacilli supplementation reduces fecal $\beta$ glucuronidase activity and affects estrogen metabolism (Baker et al., 2017). Findings from a previous study showed the correlation of IGF-1 with EGF. It is 
interesting to explore the role of yogurt or Lactobacilli supplementation in EGF toward the skin.

The type of milk that is used for fermentation might determine the nutrient content, and its fermented content results will also be correlated with EGF. Besides cow milk, the use of soybean (Glycine max) for fermented milk also widely known. Soybean contains a high number of polyphenols and phytoestrogen, such as isoflavone (Rizzo and Baroni, 2018). Isoflavone induces high expression of EGFR, thereby improving wrinkle reduction and collagen stimulation (Barret et al., 2013; Zhang et al., 2015).

Various combinations of fermented milk with probiotics have been known to have the ability to increase the surface area of villi and villus height of the intestine, thus increasing intestinal absorptive function (Adriani et al., 2019). Probiotics are living microorganisms in the gastrointestinal tract, which give benefits to the host (Appukutty et al., 2015). Formula and bacteria type for fermenting cow milk is different from soy milk. Lactic acid bacteria are used for fermentation because they easily convert lactose into glucose and galactose of cow milk. Other bacteria are also commonly used for fermenting milk, such as Lactobacillus bulgaricus and Lactobacillus acidophilus (Lengkey and Adriani, 2009). Some products replace Lactobacillus bulgaricus with Bifidobacterium spp. due to the difference in metabolism products. Bifidobacterium spp. produces the isoform of $\mathrm{D}-(+)$-lactic acid, which has an active role in physiology. Meanwhile, Lactobacillus bulgaricus produces D-(-)-lactic acid, which is physiologically inactive (Blanpain and Fuchs, 2009). The presence of Streptococcus thermophiles can enhance the digestion of lactose through the activity of its microbial $\beta$-galactosidase (Yu et al., 2020).

Due to the reasons mentioned above, a combination of Bifidobacterium spp and Lactobacillus acidophilus has shown the potential to increase the activity of lipase and protease (Lengkey and Adriani, 2010). Fermented milk formulation of Lactobacillus acidophilus and Bifidobacterium spp. also increase the non-pathogenic population and decrease the pathogenic bacteria in the colon (Adriani and Lengkey, 2010). On the other hand, adding beneficial microorganisms to the gut could prevent or delay some age-associated diseases by improving the immune response or by producing bioactive metabolites. Unfortunately, there is limited information about the effect of formulated fermented cow milk and soy milk on skin physiological homeostasis, specifically via EGF.

Thus, in this present study, we want to explore possible different effects from probiotics composition and isoflavone from soya on the epidermal thickness and EGF protein level in female Wistar rats.

\section{MATERIALS AND METHODS}

Fermented soy milk and cow milk: Four different formulations of fermented soy milk and cow milk were obtained from the Faculty of Animal Husbandry, Universitas Padjadjaran. The formulations were given with the dosage of $1.25 \%$ of body weight. For each milliliter, the formulation contains $10^{7}$ probiotics. The fermentation process is started by heating the milk at 85 ${ }^{\circ} \mathrm{C}\left(185^{\circ} \mathrm{F}\right)$. After heating, the milk is allowed to cool to $45^{\circ} \mathrm{C}\left(113^{\circ} \mathrm{F}\right)$. The bacterial culture is then combined, and a temperature of $45{ }^{\circ} \mathrm{C}$ is maintained for 4 to 12 hours to allow fermentation to occur. Four formulation type were used in this study were shown in Table 1.

Table 1. Composition of probiotics used in the study.

\begin{tabular}{|c|c|c|c|}
\hline No & Group & Composition & Probiotics \\
\hline 1 & P0 & $\begin{array}{l}\text { Control } \\
(\mathrm{NaCl} \mathrm{0,9 \% )}\end{array}$ & Water \\
\hline 2 & $\mathrm{P} 1$ & Cow Milk & $\begin{array}{l}\text { Lactobacillus bulgaricus } \\
\text { Streptococcus } \\
\text { thermophiles } \\
\text { Lactobacillus acidophilus }\end{array}$ \\
\hline 3 & $\mathrm{P} 2$ & $\begin{array}{l}\text { Cow Milk } \\
75 \%+ \\
\text { Soymilk } \\
25 \%\end{array}$ & $\begin{array}{l}\text { Lactobacillus bulgaricus } \\
\text { Streptococcus } \\
\text { thermophiles } \\
\text { Lactobacillus acidophilus }\end{array}$ \\
\hline 4 & P3 & $\begin{array}{l}\text { Cow Milk } \\
50 \%+ \\
\text { Soymilk } \\
50 \%\end{array}$ & $\begin{array}{l}\text { Lactobacillus bulgaricus } \\
\text { Streptococcus } \\
\text { thermophiles } \\
\text { Lactobacillus acidophilus }\end{array}$ \\
\hline 5 & P4 & Cow Milk & $\begin{array}{l}\text { Lactobacillus acidophilus } \\
\text { Bifidobacterium bifidum }\end{array}$ \\
\hline
\end{tabular}

Animal treatment: The treatment given to animals included handling, maintenance, and euthanasia and were performed after approval by the Ethics Committee, Faculty of Medicine, Universitas Padjadjaran, with registration number 1088/UN6.KEP/EC/2019. This experiment used 25 female Wistar rats with aged 12 weeks. Before experimenting, the rats were bred in the Animal Facility of PT Bio Farma, Indonesia. The rats were kept at a temperature of $24{ }^{\circ} \mathrm{C}$ with a 12-hour dark and light cycle. Food and water were given ad libitum in Animal Laboratory, Physiology Division, Faculty of Medicine, Universitas Padjadjaran.

The rats were randomly divided into the control and treatments group. Four different formulations of fermented soy milk and cow milk were given to the treatment group for 12 weeks, and water was given to the control group by oral gavaging. The dose administered to the treatment group was $1.25 \%$ of body weight and were given into four groups $\left(\mathrm{P}_{1}, \mathrm{P}_{2}, \mathrm{P}_{3}\right.$, and $\left.\mathrm{P}_{4}\right)$ based on the formulation. Treatment and water were given daily every 
morning at the same time for 12 weeks. For the control group, a standard diet is given. The rats were sacrificed using a carbon dioxide chamber (hypoxic). The dorsal skin was obtained, weighed, and rapidly frozen in liquid nitrogen and stored at $-80{ }^{\circ} \mathrm{C}$ until used for analysis.

Protein extraction and western blot: Twenty-five milligrams of each sample were taken in an Eppendorf tube. Ice cold lysis buffer $(300 \mu \mathrm{L})$ was then added rapidly to the tube. The samples were homogenized and centrifuged at $12,000 \mathrm{rpm}$ at $4{ }^{\circ} \mathrm{C}$. The supernatant was then aspirated and stored before being analyzed by western blot. The proteins $(10 \mu \mathrm{L})$ were then separated on SDS-PAGE and transferred to a nitrocellulose membrane (GE Healthcare) by electrophoresis for 150 minutes. A blocking reagent $(2 \%$, GE Healthcare) was added to the membrane and incubated at $4{ }^{\circ} \mathrm{C}$ overnight. Primary antibodies: EGF (Ab77851, Abcam), and $\beta$-actin (MA515739, Thermo Scientific) was used for immunoblotting. Visualization of the membrane was done using a chemiluminescence reagent (GE Healthcare) and read by LI-COR C-DiGit Chemiluminescence Western Blot Scanner. Quantification was done using ImageJ software $(\mathrm{NIH})$. The $\beta$-actin antibody was used as the internal control for protein levels monitoring.

Histological analysis: The skin samples were cut into about $0.3 \times 0.4 \times 0.5 \mathrm{~cm}$ in size and put into the formaldehyde solution for 24 hours. After dehydration, the samples were set into paraffin block and cut by a microtome. The samples are then rehydrated and stained using Hematoxylin and Eosin. The observed skin tissue specimen was examined and taken by the Axiocam MR3 microscope camera. The image then reviewed in ZEN 2 PRO software.

Statistical analysis: Data were analyzed by One-Way Analysis of Variance (ANOVA) test using SPSS version 20.0 software for Windows. Data were presented as means \pm standard error of the mean (means \pm SEM). Significant results are defined by a p-value $<0.05$.

\section{RESULTS}

Stool length and weight between groups of rats: Stools were collected, weighed, and measured from both groups. A decreasing pattern was found in stool weight with P1 having the largest weight and progressively decreasing to P4. Stool length for the P3 group was also decreased compared to the control group. There was an increased length of stool in P4 (Figure 1). We analyzed the stool length and weight to explore whether the treatment affected the gross morphology of the stool through changes in gut microbial diversity. The stools of the rats showed observable differences in appearance, consistency, size, color, and smell, as shown in Figure 1. The control group showed larger, paler, and harder stool, compared to the smaller, darker, and softer stool of the treatment group.
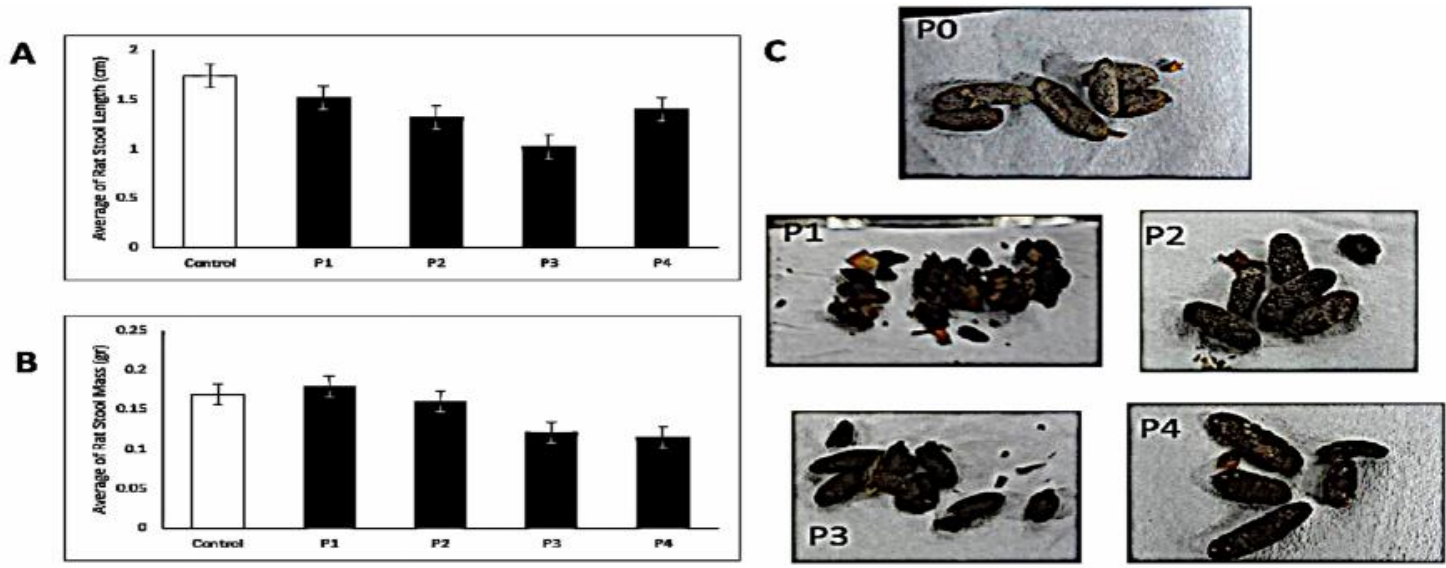

D
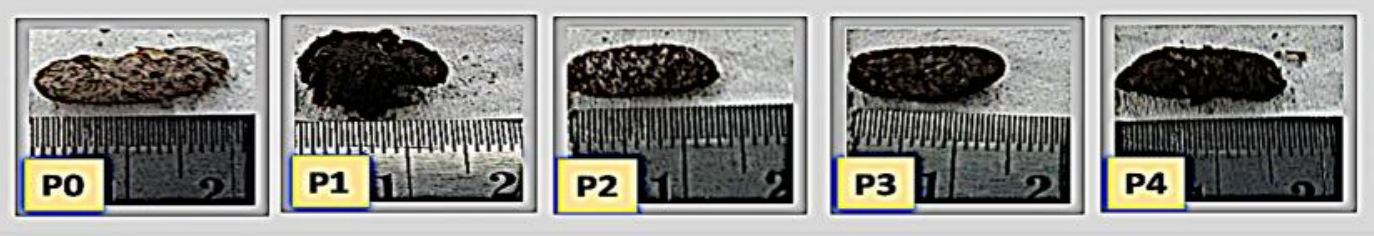

Figure 1. The different formula of fermented cow and soy milk affects rat faeces length, weight, and color after 12 weeks of treatment. Data were represented as average mean \pm SEM, (A) average length of stool, (B) average weight of feces, $(\mathrm{C})$ Changes of the stool color and length 
Cow milk fermented by Bifidobacterium bacterium increased epidermal thickness the most: Skin samples were taken from the dorsum of rats. The skin was collected, weighed, and processed as a histology specimen. Epithelial cells can be found regularly in the epidermis. The epidermis was denser and thicker than the other layers. The dermis can be divided into two layers, the papillary and reticular dermis. The papillary dermis is located between the reticular dermis and the epidermis. Generally, the papillary dermis contains more cells than the reticular layers, while the reticular layers contain a denser structure of connective tissue. The collagen fibers and elastic fibers of the reticular layer stain stronger with intense color. There were differences in epidermal thickness among the different formulations, as shown in Figure 2.

A

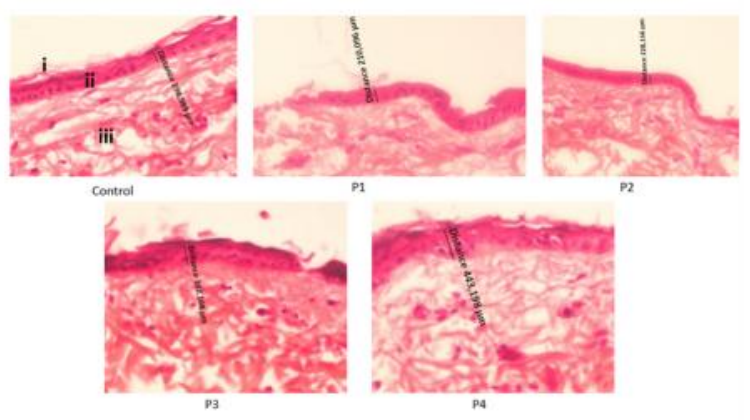

B

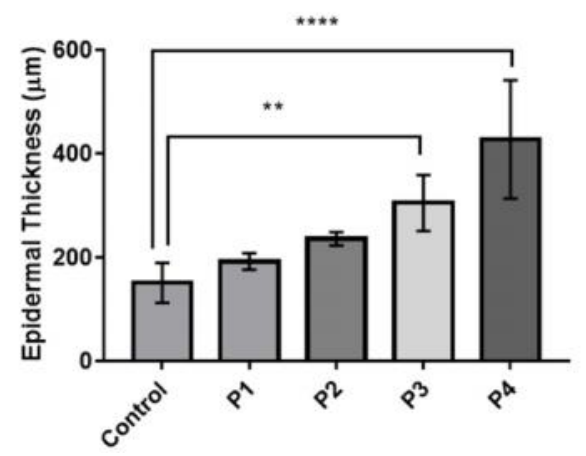

Figure 2 Effect of different formulation of fermented milk and soymilk on rat epidermal thickness (A). The representative of the histological section of each group (i) Stratum corneum. (ii) epidermis. (iii) dermis. (B). The differences in average of epidermal thickness. Data were represented in average mean \pm SEM with ${ }^{* *} \mathbf{P}<0.01 ;{ }^{* * *} \mathbf{P}<0,0001$.

The average epidermal thickness of all the treatment groups was higher than the control group. This phenomenon may reflect the direct effect of EGF on the rate of epidermal proliferation. It was shown that higher percentage of soy milk was directly proportional to the epidermal thickness. However, the formula of
Bifidobacterium bifidum showed the highest epidermal thickness regardless of the presence of isoflavone. We analyzed the epidermal thickness to explore the significant phenotype differences that are affected by EGF.

EGF was increased the most by the formulation of Bifidobacterium bifidum: Analyzing the protein level of EGF may correlate with epidermal thickness increase from the control group to the $\mathrm{P} 4$ group. There was a parallel increase in EGF protein level and epidermal thickness, as shown in Figure 3. There was a rise in epidermal thickness from control to $\mathrm{P} 4$ in a sequential manner, as shown in Figure 2. Probiotics exhibited the potential to increase the level of EGF and, therefore, epidermal proliferation. Soy milk enhances the properties, as shown by the greater increase in epidermal thickness by the $50 \%$ soy milk compared to the $25 \%$ soy milk. Comparing bacterial content, Bifidobacterium bifidum showed the greatest effect compared to Lactobacillus bulgaricus, Streptococcus thermophiles, and Lactobacillus acidophilus even though there is an absence of soymilk in this treatment groups.

A

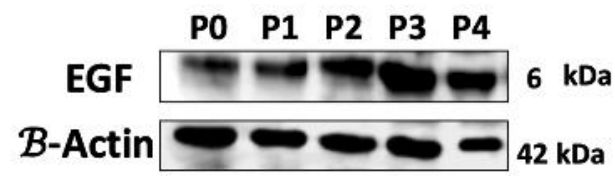

B

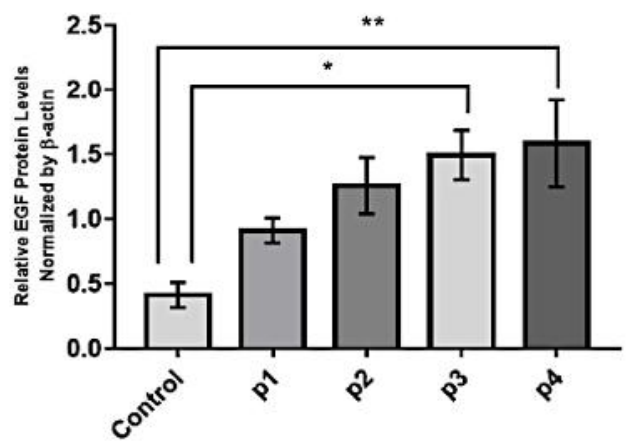

Figure 3. The increase of epidermal thickness and EGF level took effect in a parallel manner. This induction stimulated epidermal proliferation in skin tissue. Representative immunoblotting was shown (A), and densitometric quantification was shown in the graph normalized by $\beta$-actin (B). Data were represented as average ratio \pm SEM with *(P $<0.05){ }^{* *}(\mathrm{P}<0.01)$.

\section{DISCUSSION}

A fermented food, such as yogurt, has been a part of our diet with potential beneficial effects Yoghurt 
or otherwise known as fermented milk, is known to be beneficial due to the live microorganism called probiotics. The most common species of probiotics belong in the families of Lactobacillus, Bifidobacterium, and Streptococcus (Lolou and Panayiotidis, 2019) with the first two families being the most studied in relation to human health (Kittibunchakul et al., 2018). However, there is a small number of studies showing the beneficial effects of probiotics on the skin. L. lactis strain H61 supplementation, daily for eight weeks, increased skin elasticity on middle-aged women (Kimoto-Nira et al., 2012). Oral intakes of L. plantarum (HY7714) on middleaged patients, increased skin moisture, decreased the depth of wrinkles, and improve skin glossiness and elasticity (Lee et al., 2015). Moreover, other studies have shown consistent results using L. rhamnosus, B. breve Strain Yakult, L. lactis, $S$. thermophilus showing improved skin hydration and serum phenol levels (Kano et al., 2013; Lee et al., 2018).

Previous findings showed a strong symbiotic relationship between microorganisms and its microbiota. This natural microflora supports the immune system in various ways, including the production of natural antimicrobial compounds (e.g., lactic acid) as well as activation of various signalling pathways and modulation of the inflammatory response (Flandroy et al., 2018; Grice, 2014). Overexpression of pro-inflammatory cytokine IL-6 tends to happen in the aging gut, which affects the inflammatory rate in aging skin (Rentini et al., 2015). The aging gut showed lower microbial biodiversity, which is associated with an increase of proinflammatory bacteria (Biagi et al., 2010). Probiotics can improve the condition of microbial dysbiosis in the aging gut and establish health-promoting strains of bacteria. Consumption of probiotics consisting of galacto oligosaccharides (GOS) increases CD44, TIMP-1, and type 1 collagen, which are the markers of dermal cell adhesion and matrix formation, thus improving the rejuvenation of the skin. GOS was able to switch unfavorable amino acid metabolism and phenols production by becoming an additional source of metabolism for the gut microbiota (Collins and Reid, 2016).

However, the correlation between probiotics contained in fermented milk toward skin regeneration, specifically epidermal homeostasis, is still limited. Epidermal homeostasis is a renewal process of the epidermis and its appendages as a replacement mechanism of lost cells and matrix during the turnover or after injury (Adriani and Lengkey, 2010). The modulation of the EGFR ligand system must be evoked to activate the downstream pathway by various ligands and initiate the skin maintenance process. The ligands are transforming growth factor- $\alpha$ (TGF- $\alpha$ ), amphiregulin (AREG), heparin-binding EGF-like growth factor (HBEGF), betacellulin (BTC), epiregulin (EREG), and epigen
(EPGN) (Blanpain and Fuchs, 2009). EGFR binding to its ligands promotes signaling of the intrinsic kinase domain, followed by specific tyrosine kinase residues phosphorylation in the cytoplasmic tail that leads to activation of multiple pathways (Nanba et al., 2013). In this study, the consumption of probiotics might improve the epidermal thickness (Figure 2). It showed their potential for maintaining the homeostasis and overcoming the effect of external factors.

One of the causative aspects of skin aging is the presence of Reactive Oxygen Species (ROS). ROS triggers the expression of matrix metalloproteinases (MMP-1, MMP-3, and MMP-9). MMPs will degrade collagen that constructs the skin tissue. The degradation process will decrease skin tension and increase the formation of more ROS. This process took place in a looping manner and is activated via the MAPK pathway (Fisher et al., 2014). MAPK pathway is also a downstream pathway of EGFR activation (Rajaram et al., 2017). EGF promotes the extracellular-signal-regulated MAPK, while the ROS induces the stress-activated MAPK (Fisher et al., 2014). Thus, the elevation of EGF can inhibit the process of collagen degradation that leads to skin aging.

In this study, we also explore the possible differential effect of fermented soy milk and cow milk in female Wistar rats. Soy isoflavones bind to the estrogenic receptor (Lambert et al., 2017). This study showed that the formulation with a higher proportion of soy milk showed greater epidermal thickness (Figure 2). These findings might reflect the fact that an increasing amount of isoflavone binds to the estrogenic receptor and therefore increase the rate of collagen synthesis (Matsuura-Hachiya et al., 2018). As the epidermis becomes thinner with aging, the process is halted by the presence of isoflavones. This antiaging effect of isoflavone shows the potential of soybean to be an alternative for preserving skin health and preventing skin aging. Conduction of cell culture experiment showed that isoflavones had increased collagen, elastin, and tissue inhibitor of metalloproteinases (TIMP) gene expression (Gopaul et al., 2012).

We observed that bacteria formulation might determine the active metabolite compound resulted by the fermentation process. For instance, Bifidobacterium bifidum increases the largest surface area, elevate the most brush border enzyme, and give the best improvement to the transport system compared to Lactobacillus bulgaricus, Streptococcus thermophiles, and Lactobacillus acidophilus (Yu et al., 2020) and produce more active metabolic products than Lactobacillus spp which initiates another signaling mechanism in tissue (Lengkey and Adriani, 2009). This study showed that the composition consisting of $100 \%$ cow milk fermented by Bifidobacterium bifidum and Lactobacillus acidophilus exhibited the thickest 
epidermis. This result was also parallel with the trends in the EGF protein level observed in this study, as shown in Figure 3. The property of Bifidobacterium bifidum in enhancing gut enzymatic activity and improving gut microbial dysbiosis may be better if the isoflavone is added by adding the soy milk to the formula. The interaction between EGF and EGFR plays an essential role in maintaining skin homeostasis, proliferation, and maintenance through various interactions of the receptor and the ligands that leads to the activation of various downstream pathways. The changes that happen in the skin can be caused by various factors, including the internal and external factors. In this research, we are proposing that probiotics with the enhancement of soy isoflavones may maintain the skin homeostasis by elevating the level of EGF.

Conclusion: The consumption of various formulation of fermented milk and fermented soy milk may maintain skin structure and delay aging in skin by elevating the level of EGF. It was shown in an experiment in rats supported by the difference in epidermal thickness. Formulation of Bifidobacterium bifidum exhibited the highest potential as a skin homeostasis modulator.

Availability of Data and Material: The corresponding author will provide the data of this research upon permission and request.

Competing Interest: The authors declare that there is no competing and financial interest.

Funding: This research was funded by Penelitian Dasar Unggulan Perguruan Tinggi 2020 number: 1827/UN6.3.1/LT/2020 from DIKTI Indonesia to Ronny Lesmana and ALG grant 2020 to Lovita Andriani.

Authors Contribution: RL, LA, ZH, are responsible for designing the experiment, treatment, analyzing and writing the manuscript, western blot and histology staining; HG, YSP, NS are animal handling analyzing, sampling and collecting data, LA and US are responsible for writing, histology study, and stool analysis

Acknowledgments: The authors would like to thank Ardo Sanjaya, Susianti, Meita Sapitri, Irfan Anis Ahmad, and Canadia Ravelita for technical assistance; Biology Activity Laboratory and Molecular Physiology Laboratory, Central Laboratory, Universitas Padjadjaran.

\section{REFERENCES}

Adriani, L. and H. Lengkey (2010). Probiotic bacteria as yoghurt starter and its implication effect to the pathogenic and non-pathogenic bacteria in mice gastrointestinal. Medicină Veterinară. 53(12): 262-266.
Adriani, L., D. Latipudin, R.L. Balia and T. Widjastuti (2019). Improvement of small intestine morphometry in broiler chicken using fermented cow and soymilk as probiotic. Int. J. Poult. Sci. 18(6): 255-259.

Appukutty, M., K. Ramasamy, S. Rajan, S. Vellasamy, R. Ramasamy and A.K. Radhakrishnan (2015). Effect of orally administered soy milk fermented with Lactobacillus plantarum LAB12 and physical exercise on murine immune responses. Benef. Microbes. 6(4): 491-496.

Baker, J.M., L. Al-Nakkash and M.M. Herbst-Kralovetz (2017). Estrogen-gut microbiome axis: Physiological and clinical implications. Maturitas, 103: 45-53.

Barrett, K.E., J. Casey, R. Holland, L.J. Wainwright and G. Jenkins (2013). Wrinkle reduction in postmenopausal women consuming a novel oral supplement: a double-blind placebo-controlled randomized study. Int. J. Cosmet. Sci. 36(1): 22-31.

Biagi, E., L. Nylund, M. Candela, R. Ostan, L. Bucci, E. Pini, J. Nikkila, D. Monti, R. Satokari, C. Franceschi, P. Brigidi and W.D. Vos (2010). Through ageing, and beyond: Gut microbiota and inflammatory status in seniors and centenarians. PLoS One. 5(5): 1-28.

Blanpain, C. and E. Fuchs (2009). Epidermal homeostasis: a balancing act of stem cells in the skin. Nat. Rev. Mol. 10(3): 207-217.

Bodnar, R.J. (2013). Epidermal growth factor and epidermal growth factor receptor: the yin and yang in the treatment of cutaneous wounds and cancer. Adv. Skin. Wound Care. 2(1): 24-29.

Collins , S., and G. Reid (2016). Distant site effects of ingested prebiotics. Nutrients. 8(9): 1-20.

Fisher, G.J., D.L. Sachs and J.J Voorhees (2014) Ageing: collagenase-mediated collagen fragmentation as a rejuvenation target. Br. J. Dermatol. 171(3): 446-449.

Flandroy, L., T. Poutahidis, G. Berg, G. Clarke, M.C. Dao, E. Decaestecker, E. Furman, T. Haahtela, S. Massart, H. Plovier, Y. Sanz, and G. Rook (2018). The impact of human activities and lifestyles on the interlinked microbiota and health of humans and of ecosystems. Sci. Total Environ. 627: 1018-1038.

Gopaul, R., H.E. Knaggs and E.D. Lephart (2012). Biochemical investigation and gene analysis of equol: A plant and soy-derived isoflavonoid with antiaging and antioxidant properties with potential human skin applications. BioFactors. 38(1): 44-52.

Grice, E.A. (2014) The skin microbiome: Potential for novel diagnostic and therapeutic approaches to 
cutaneous disease. Semin. Cutan. Med. Surg. 33(2): 98-103.

Kano, M., N. Masuoka, C. Kaga, S. Sugimoto, R. Iizuka, K. Manabe, T. Sone, K. Oeda, C. Nonaka, K. Miyazaki and F. Ishikawa (2013). Consecutive intake of fermented milk containing bifidobacterium breve strain yakult and galactooligosaccharides benefits skin condition in healthy adult women. Biosci. Microb. Food Health. 32(1): 33-39.

Kimoto-Nira, H., R. Aoki, K. Sasaki, C. Suzuki and K. Mizumachi (2012). Oral intake of heat-killed cells of lactococcus lactis strain h61 promotes skin health in women. J. Nutr. Sci. 1: 1-13.

Kittibunchakul, S., T. Maischberger, K.J. Domig, W. Kneifel, H.M. Nguyen, D. Haltrich and T.H. Nguyen (2018). Fermentability of a novel galacto-oligosaccharide mixture by Lactobacillus spp. and Bifidobacterium spp. Molecules. 23(12): 1-19.

Lambert, M., L.M. Hu and P.B. Jeppesen (2017). A systematic review and meta-analysis of the effects of isoflavone formulations against estrogen-deficient bone resorption in peri- and postmenopausal women. Am. J. Clin. Nutr. 106(3): 801-811.

Lee, D.E., C.S. Huh, J. Ra, L.D. Choi, J.W. Jeong, S.H. Kim, J.H. Ryu, Y.K. Seo, J.S. Koh, J.H. Lee, J.H. Sim and Y.T Ahn (2015). Clinical evidence of effects of Lactobacillus plantarum HY7714 on skin aging: A randomized, double blind, placebo-controlled study. J. Microbiol. Biotechnol. 25(12): 2160-2168.

Lee, J.B., J.H. Suk and S.M. Kang (2018). Effect of Lactobacillus rhamnosus KCTC 5033 on the appearance of facial skin due to the ingestion of probiotics and paraprobiotics. J. Investig. Cosmetol. 14: 287-296.

Lengkey, H.A.W and L. Adriani (2009). Effects of milk fermented with Lactobacillus acidophilus and Bifidobacterium spp., on lactic acid and acetic acid content and on Staphylococcus aureus and Pseudomonas aeruginosa. Biotechnol. Anim. Husb. 25(5-6-2): 719-724.

Lengkey, H.AW and L. Adriani (2010). Implication effect of probiotic bacteria to yoghurt quality and enzyme activities. Medicină Veterinară. 53(12): 259-261.
Lolou, V., ang M.I. Panayiotidis (2019). Review: Functional role of probiotics and prebiotics on skin health and disease. Fermentation. 5(41): 117.

Matsuura-Hachiya, Y., K.Y. Arai, T. Muraguchi, T. Sasaki and T. Nishiyama (2018). Type IV collagen aggregates promote keratinocyte proliferation and formation of epidermal layer in human skin equivalents. Exp. Dermatol. 27(5):443-448.

Nanba, D., F. Toki, Y. Barrandon and S. Higashiyama (2013). Recent advances in the epidermal growth factor receptor/ligand system biology on skin homeostasis and keratinocyte stem cell regulation. J. Dermatol. Sci. 72(2): 81-86.

Rajaram, P., P. Chandra, S. Ticku, B.K Pallavi, K.B. Rudresh and P. Mansabdar (2017). Epidermal growth factor receptor: Role in human cancer. Indian J. Den. Res. 28(6): 687-694.

Rentini, S., M. Marini, M. Regoli, C. Nicoletti, E. Bertelli, A.J.M Watson and C. Nicoletti (2015). Age-associated modifications of intestinal permeability and innate immunity in human small intestine. Clin. Sci. 129(7): 515-527.

Rizzo, G. and L. Baroni (2018). Soy, soy foods and their role in vegetarian diets. Nutrients. 10(1): 1-51.

Srinivasan, V., K. Nimptsch and S. Rohrmann (2019). Associations of current, childhood, and adolescent milk intake with serum insulin-like growth factor (IGF)-1 and IGF binding protein 3 concentrations in adulthood. Nutr. Cancer. 71(6): 931-938.

Wang, Z. (2017). ErbB receptor signaling. 1st Ed. Springer; Berlin (Germany). $303 \mathrm{p}$

Yu, P., N. Li, M. Geng, Z. Liu, X. Liu, H. Zhang, J. Zhao, H. Zhang and W. Chen (2020). Short communication: Lactose utilization of Streptococcus thermophilus and correlations with beta-galactosidase and urease. J. Dairy Sci. 103(1): 166-171.

Zeng, F. and R.C. Harris (2014). Epidermal growth factor, from gene organization to bedside. Semin. Cell Dev. Biol. 28: 2-11.

Zhang, Y., S.K.C Chang and Z. Liu (2015). Isoflavone profile in soymilk as affected by soybean variety, grinding, and heat-processing methods. J. Food. Sci. 80(5): C983-C988. 